\title{
Isometries, Tessellations and Escher, Oh My!
}

\author{
Melissa Potter and Jason M. Ribando \\ Department of Mathematics \\ University of Northern lowa \\ Cedar Falls, lowa 50614-0506 USA
}

Received: August 14, 2003 Accepted: December 14, 2004

\section{INTRODUCTION}

Most people are familiar with tilings of the Euclidean plane. They give rise to the seventeen distinct wallpaper patterns that pervade bathroom floors and parlor walls. However, the Euclidean plane is not the only space that can be tessellated; nonEuclidean geometries also have tiling patterns. Those familiar with hyperbolic tessellations probably know them via the wonderful artistry of M.C. Escher.

In this paper we consider the isometries of the hyperbolic plane as a means for analyzing hyperbolic tessellations. We use a recent article by Chaim Gooodman-Strauss to make the connection between hyperbolic tessellations and their constructions using Euclidean tools. Understanding the isometries of the hyperbolic plane and their synthetic constructions provides a deeper appreciation of the hyperbolic tessellations illustrated by M.C. Escher in his Circle Limits I-IV prints.
\end{abstract}

ABSTRACT

Motivated to better understand the wonderful artistry and hyperbolic tessellations of M.C. Escher's Circle Limit prints, we study the isometries of the hyperbolic plane and create tessellations of the Poincaré disk using the Euclidean tools of compass and straightedge.

\section{THE HYPERBOLIC PLANE}

We begin with a word from our sponsor, the hyperbolic plane. In 1868 Eugenio Beltrami provided the first model, the pseudosphere, for an incidence geometry agreeing with Euclid's first four postulates while violating the fifth [7]. Today, Poincaré's disk model is more frequently used to explore hyperbolic geometry, which does not assume the parallel postulate. In this non-Euclidean geometry, for a given line $I$ and a point $P$ not on I, there exist infinitely many lines containing $P$ and not intersecting I. The points of the disk model are interior to the unit disk; points on the boundary are called points at infinity and do not properly belong to the hyperbolic plane. We use $\mathrm{H}$ to denote the points in the hyperbolic plane and $\partial \mathrm{H}$ to denote the boundary points. For the purposes of considering transformations

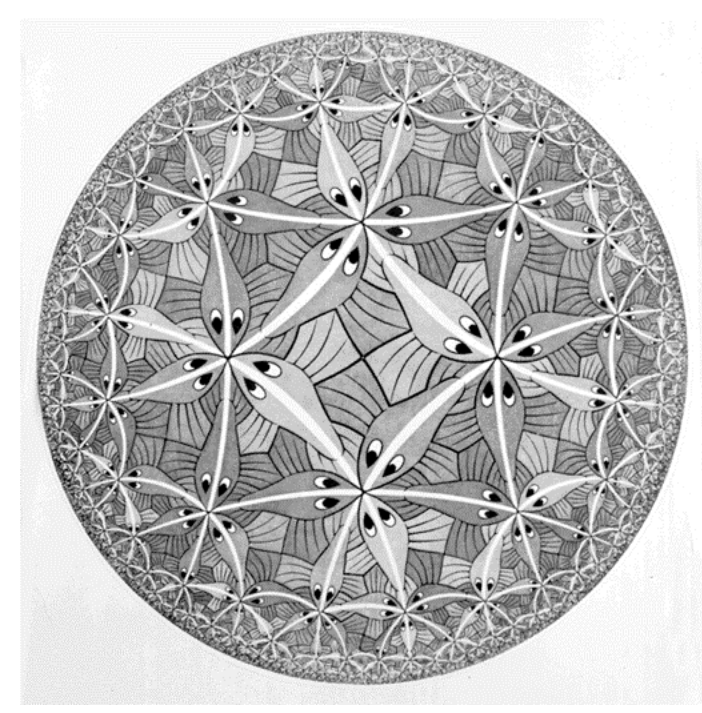

Figure 1. M.C. Escher's "Circle Limit III" (C) 2004 The M.C. Escher Company - Baarn - Holland. All rights reserved. 
and constructions later, it may be useful to think of the Poincaré disk as being embedded in the Euclidean plane. The lines of $\mathrm{H}$ are arcs of Euclidean circles that meet $\partial \mathrm{H}$ at right angles. As with any geometry in which distance has meaning, these shortest distance paths are called geodesics. Hyperbolic geodesics also include diameters of the disk, which are taken to be Euclidean circles of infinite radius.

Given a geodesic I and a point $P$ not on I in $\mathrm{H}$, there are infinitely many hyperbolic geodesics containing $P$ that don't intersect $I$. Two geodesics in $\mathrm{H}$ intersect, are parallel, or are ultra-parallel according to whether they contain a common point, meet at a point at infinity, or don't meet at all. Figure 2 illustrates the Poincaré disc with a pair of geodesics meeting at $\mathrm{P}$, a pair of ultraparallels meeting at a point at infinity, and a remaining pair of parallel lines.

\section{ISOMETRIES}

An isometry, sometimes called a rigid motion, is a distance preserving transformation that maps a space onto itself. In the Euclidean plane there are three basic isometries: axial reflections, rotations, and translations. According to the fundamental theorem of geometric transformations, any isometry of the Euclidean plane is the composition of one, two or three axial reflections. A rotation by $\theta$ radians about a point $P$, for example, is equivalent to the composition of reflections about two lines intersecting at $P$ and forming an acute angle of $\theta / 2$.

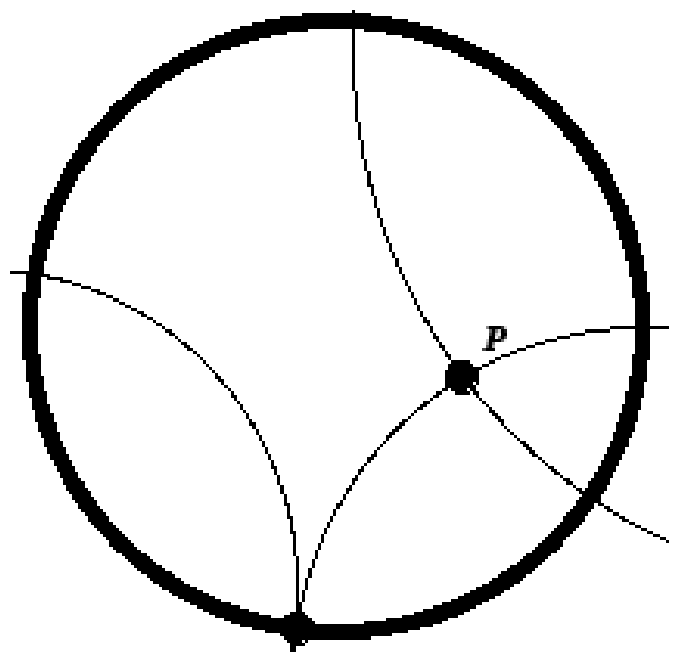

Figure 2. Hyperbolic geodesics.
There are four basic isometries in the hyperbolic plane: non-Euclidean reflection, non-Euclidean rotation, nonEuclidean translation, and parabolic isometry. The first three have the obvious analogues in the Euclidean plane. As with Euclidean isometries, an isometry in the Poincaré disk is the composition of at most three non-Euclidean reflections, although the four basic isometries require at most two. Moreover, the image of any point in the Poincaré disk under a hyperbolic isometry can be constructed with a Euclidean compass and straightedge by making use of circle inversions.

A circle inversion in the Euclidean plane maps points in the interior of a circle to its exterior, maps points exterior to the circle to its interior, and leaves points on the circle invariant. More formally, we define the circle inversion about a circle $\mathrm{C}$ centered at point $X$ with radius $r$ as follows. It is the transformation that maps any point $W$ in the Euclidean plane, excluding the center of the circle $X$, to the unique point $Z$ such that $X, W$ and $Z$ are collinear and

$$
X W \cdot X Z=r^{2} .
$$

Note that circle inversion about $C$ cannot act on the center of the circle. As the distance from the center of the circle to itself is zero, the above equation cannot be satisfied. Some mathematicians patch the definition by adding a point at infinity to the plane and call this union the extended plane. Circle inversion on the extended plane swaps the center of the circle with this distinguished point.

Thus far we have defined circle inversion as a transformation on the Euclidean plane, but which circle inversions result in isometries of the Poincaré disk? The answer is that inversions over circles that intersect $\partial \mathrm{H}$ orthogonally map points inside $\mathrm{H}$ to points inside $\mathrm{H}$. As arcs of circles that meet the boundary orthogonally are geodesics in $\mathrm{H}$, circle inversions about such circles in $\mathrm{H}$ are analogous to axial reflections in the Euclidean plane. Thus they are called non-Euclidean reflections. In the limiting case where the circle of inversion has infinite radius and is a diameter of $\mathrm{H}$, a circle inversion acts on the points of $\mathrm{H}$ just as a Euclidean axial reflection does. 
It is also interesting to note that just as lines separate the Euclidean plane into two half planes, hyperbolic geodesics separate $\mathrm{H}$ into two halves. However, these half planes may not appear to represent equal halves by Euclidean standards because hyperbolic distances are distorted in the disk model.

The classical tools of Euclidean geometry-the compass and straightedgecan be used to find the image of a point under non-Euclidean reflection in $\mathrm{H}$ the same way that they are used to find the image of a point under circle inversion in the Euclidean plane. This fact is particularly remarkable considering we can use the same synthetic construction in two different geometries. Suppose we wish to find the image $Z$ of a point $W$ by non-Euclidean reflection about a geodesic $\mathrm{I}$. Let $\mathrm{C}$ be the Euclidean circle containing $I$, and let $X$ be its center. There are three cases to consider according to the position of $\mathrm{W}$ relative to $\mathrm{C}$. If $W$ lies on $C$, then $W$ remains invariant by non-Euclidean reflection about I and $Z=W$. If $W$ does not lie on $C$, construct the (Euclidean) line containing $X$ and $W$. If $W$ is in the interior of $\mathrm{C}$, construct the right triangle configuration as shown in Figure 3. Here, $Y$ is a point on $I$ and on the line perpendicular to $\overleftrightarrow{X W}$ at $\mathrm{W}$. The image point $Z$ is the intersection of the tangent to $C$ at $Y$ and $\overleftrightarrow{X W}$. If $\mathrm{W}$ lies exterior to $\mathrm{C}$, the same figure applies after interchanging the roles of W and $Z$, though the construction steps vary slightly.

To show that $Z$ is in fact the image of $W$ after an inversion over the circle centered at $\mathrm{X}$, consider the three similar right triangles $\triangle \mathrm{XWY}, \triangle \mathrm{XYZ}$ and $\triangle \mathrm{YWZ}$. The similarity relations imply

$$
\frac{X W}{X Y}=\frac{X Y}{X Z}
$$

As $X Y$ is the radius of the circle of inversion, we have

$$
(X Y)^{2}=r^{2}=X W \cdot X Z .
$$

Thus, this construction upholds the definition of circle inversion.

What about the other basic isometries of $\mathrm{H}$ ? A non-Euclidean translation is the composition of circle

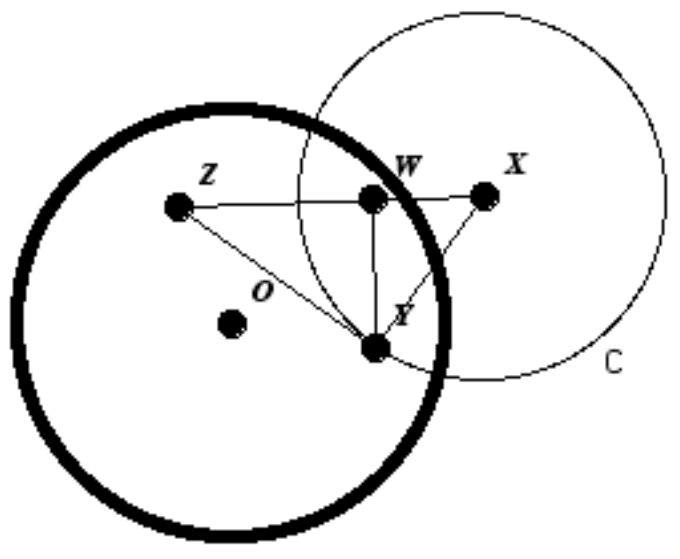

Figure 3. Non-Euclidean reflection.

inversions about two ultra-parallels. In $\mathrm{H}$ every pair of ultra-parallels has a unique Euclidean circle perpendicular to both, and a non-Euclidean translation occurs along this common orthogonal circle. This isometry resembles a translation in Euclidean space as points appear to slide across the disk along the common orthogonal line. In Figure 4, the non-Euclidean translation maps point $P$ to $R$ via $Q$.

Non-Euclidean rotation is the composition of two non-Euclidean reflections about geodesics meeting at a point in $\mathrm{H}$. This isometry resembles a Euclidean rotation as points move around the disk along hyperbolic circles centered at the point of intersection. The magnitude of the rotation, just as in Euclidean space, equals twice the acute angle formed at the intersection of the two lines. The nonEuclidean rotation illustrated in Figure 5 maps $P$ to $R$ via $Q$.

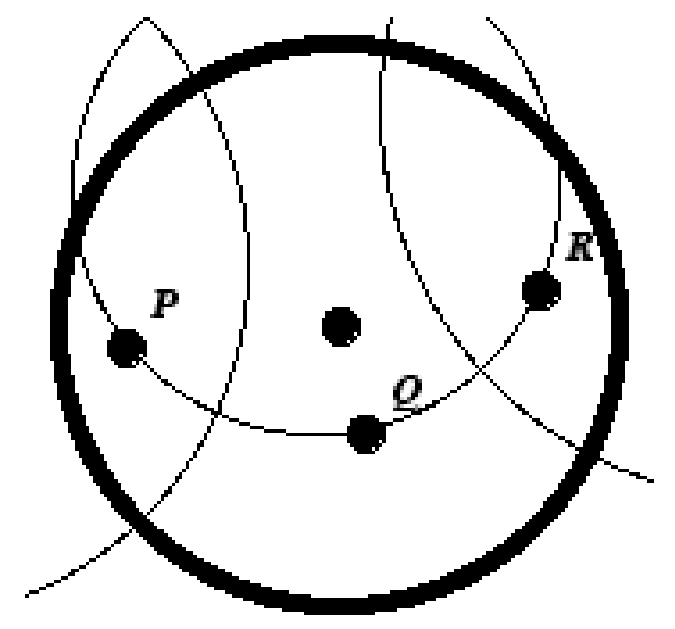

Figure 4. Non-Euclidean translation. 


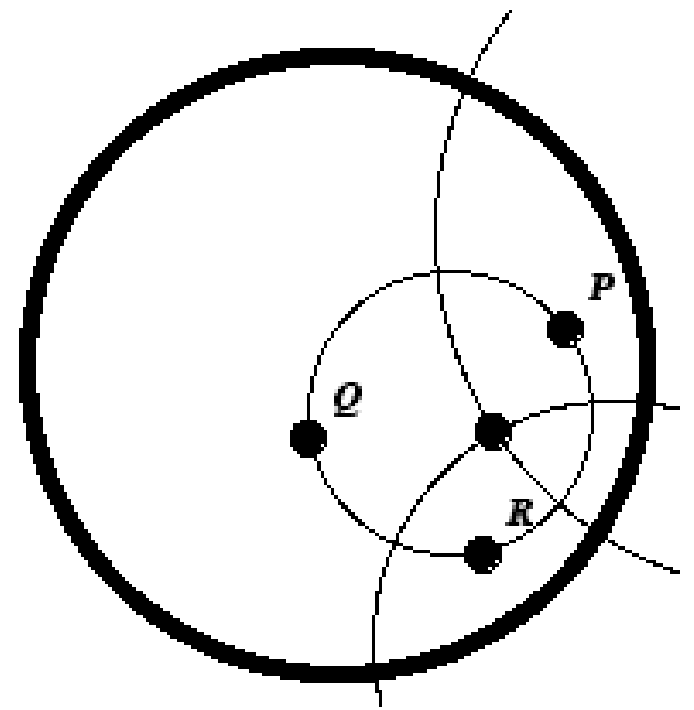

Figure 5. Non-Euclidean rotation.

Parabolic isometry is a limiting case of both non-Euclidean rotation and nonEuclidean translation and has no true Euclidean counterpart. Suppose two parallels $\overleftrightarrow{A B}$ and $\overleftrightarrow{A^{\prime} B}$ share a common boundary point $\mathrm{B}$. In this case points in $\mathrm{H}$ are transformed as if they are rotated about the point $B$ at infinity. Under a nonEuclidean rotation, points in $\mathrm{H}$ move along a horocycle, a hyperbolic circle whose center is a point at infinity. However, as no point in $\mathrm{H}$ remains fixed, parabolic isometry is not strictly analogous to Euclidean rotation. If one allows the boundary point $B$ to migrate to the interior of $\mathrm{H}$, the composition of the non-Euclidean reflections about $\overleftrightarrow{A B}$ and $\overleftrightarrow{A^{\prime} B}$ becomes a non-Euclidean rotation. Likewise, if the common boundary point $B$ resolves into two boundary points $\mathrm{B}$ and $\mathrm{B}^{\prime}$ such that $\overleftrightarrow{A B}$ and $\overleftrightarrow{A^{\prime} B^{\prime}}$ don't intersect in $\mathrm{H}$, the composition of the non-Euclidean reflections becomes a non-Euclidean translation. The parabolic isometry in Figure 6 maps $\mathrm{P}$ to $\mathrm{R}$ via $\mathrm{Q}$.

\section{TESSELLATIONS}

As in the Euclidean plane, combinations of hyperbolic isometries give rise to beautiful tessellations. Although mathematicians and artists have been constructing and investigating hyperbolic isometries for years, it was not until recently that the process of constructing a tessellation of $\mathrm{H}$ was carefully recorded.
In the January 2001, issue of the American Mathematical Monthly, Chaim Goodman-Strauss published "Compass and Straightedge in the Poincaré Disk," which "may be the first detailed, explicit synthetic construction of triangle tilings of the Poincaré disk to appear in the mathematical literature" [5]. In his article GoodmanStrauss demonstrates ten elementary constructions such as inverting a point through a circle and constructing geodesics through two points in the disk. Once the reader has mastered those constructions, he or she can progress to constructing tessellations of the hyperbolic plane.

Goodman-Strauss's method for constructing tessellations of the hyperbolic plane is surprisingly similar to the method used for constructing a Euclidean tessellation. In the Euclidean plane we typically start with a polygon and then repeatedly reflect the polygon over its edges or perform some other isometry to the figure to cover the plane. In the hyperbolic plane, we also begin by constructing a polygon, in our case a hyperbolic triangle, and then repeatedly invert the triangle over its edges to extend the tiling. However, because hyperbolic line segments in $\mathrm{H}$ are defined as portions of circular arcs, it is almost as difficult to construct the appropriate base triangle as it is to construct the subsequent triangles.

To keep things simple, GoodmanStrauss states that a hyperbolic tessellation is defined by "an unordered triple of whole

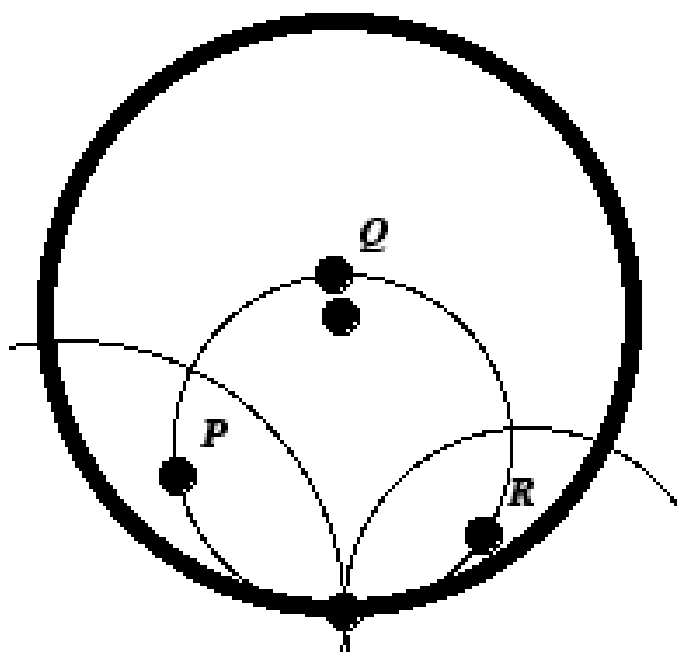

Figure 6. Parabolic Isometry. 
numbers $p, q, r$ with

$$
\frac{1}{p}+\frac{1}{q}+\frac{1}{r}<1 .
$$

There is a tiling of the hyperbolic plane by triangles with interior angles $\pi / p, \pi / q, \pi / r$, generated by inversions across the sides of some base triangle" [5]. In his paper he first constructs the 7, 4, 2 triangle tiling (Figure 7) and then proceeds to describe the steps for constructing any tiling of the form $p, q, 2$ such that

$$
\frac{1}{p}+\frac{1}{q}<\frac{1}{2} .
$$

In his construction of the 7, 4, 2 tiling, Goodman-Strauss begins by assuming the construction of a regular septagon. Replicating his construction is difficult because a regular septagon is not constructible with compass and straightedge alone; it requires a notched straightedge. Given a regular septagon centered at $\mathrm{O}$, construct the lines that form $\triangle$ OVB with any vertex $V$ of the septagon and the midpoint $B$ of an adjacent side. Construct a circle $\mathrm{C}$ centered at the midpoint $\mathrm{P}$ of $\overline{O B}$ with radius OP and a circle $D$ centered at $B$ with radius $B V$. We will take as the boundary of our Poincaré disk the circle centered at $\mathrm{O}$ passing through the intersection points of $\mathrm{C}$ and $D$.

Let $Q$ be the second intersection point of the diameter $\overline{O V}$ with circle $\mathrm{D}$. Then hyperbolic $\triangle \mathrm{OPQ}$ is a right triangle with angles $\pi / 7, \pi / 2, \pi / 7$. Bisecting $\triangle \mathrm{OPQ}$ with an altitude to $\mathrm{P}$ (along a geodesic) produces hyperbolic $\triangle$ OPR with angles $\pi / 7$, $\pi / 4$, and $\pi / 2$ (see Figure 8 ). From here we could repeatedly invert the hyperbolic $\triangle$ OPR over its edges. However GoodmanStrauss suggests taking advantage of the dihedral symmetry of the septagon and now repeating the previous steps with another midpoint of a septagon edge. Keeping in mind that one can always revert to circle inversions if need be, Goodman-Strauss provides further shortcuts for filling in the remaining portions of the disk that bypass repeated inversions.

Goodman-Strauss refers to the method of inverting points across lines to create more versions of the base triangle "the crudest, but most general (and clearly

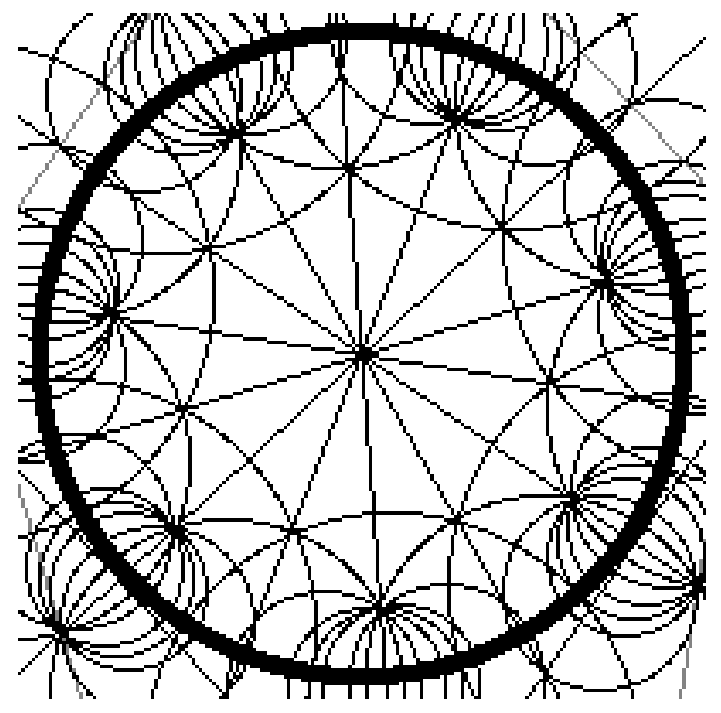

Figure 7. The 7, 4, 2 tiling.

most successful) method" of tessellating $\mathrm{H}$ [5]. However, Goodman-Strauss was working with paper and pencil. When working with geometry software, such as Cinderella $^{\mathrm{TM}}$, that will automatically invert points and construct circles through three non-collinear points, it is much easier to use the "crude" method. Figures 9 and 10 illustrate tessellations of the hyperbolic plane the authors created using GoodmanStrauss's techniques and Cinderella ${ }^{\mathrm{TM}}$.

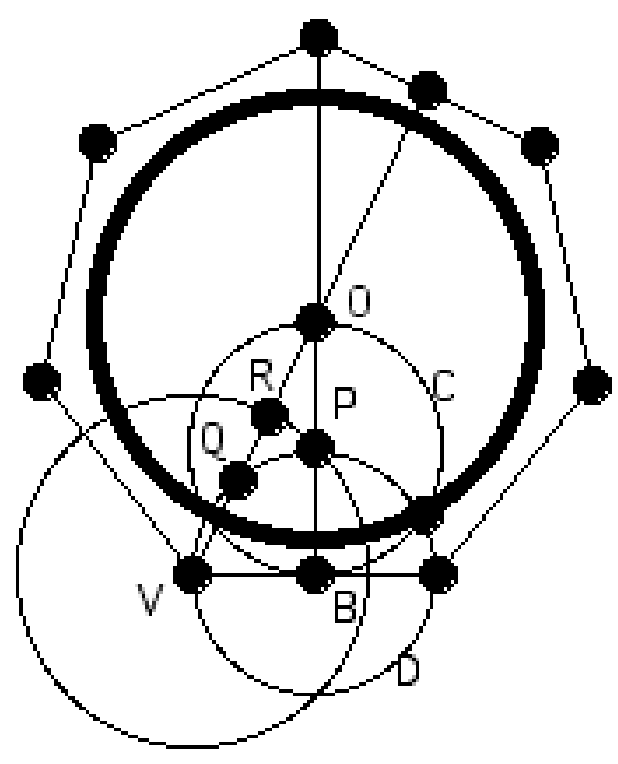

Figure 8. The $7,4,2$ base $\triangle \mathrm{OPR}$. 


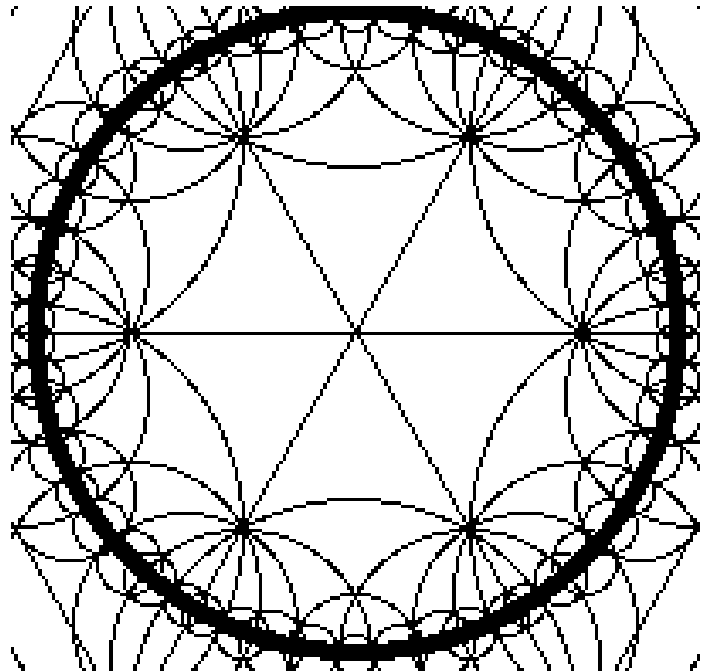

Figure 9. Tessellation of the hyperbolic plane by $60-30-30$ triangles.

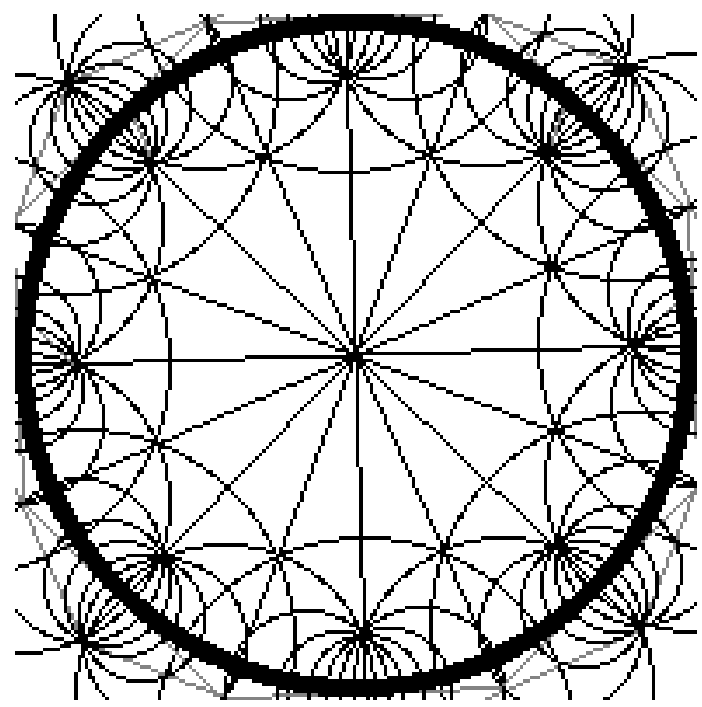

Figure 10. Tessellation using $8,4,2$ triangles.

\section{ESCHER'S CIRCLE LIMIT PRINTS}

M. C. Escher, the famed Dutch graphic artist, is best known for his artistic tessellating patterns. Early in his career he drew Euclidean tessellations, because that was all he was familiar with, but he often wondered how he could create new and different types of repeating patterns. $\mathrm{He}$ once mentioned "It's logical that, by gradually reducing each figure, a limit of the infinitely small can theoretically be reached, symbolizing an infinity of number, or a totality. This idea has long fascinated me" [4]. Escher attempted to achieve this infinite number of figures, but was often dissatisfied with his efforts because the tessellated images would never end, as they grew larger toward the edge of his canvas. Thus they lacked the totality he sought. He was able to create a satisfactory limiting work in Square Limit but soon pondered the possibility of limiting space with a circular boundary. Escher once said that he knew that a circle limit "is no simple question, but a complicated, non-Euclidean problem, much too difficult for a layman, as I am. After a long time of trying, in vain, in my own experimental way, I was finally put on the right path by a publication of the English mathematician Professor H. S. M. Coxeter" [4].

Escher became a lifelong friend of Coxeter when the two met at the international Congress of Mathematicians in 1954. A few years after this, Coxeter used some of Escher's tessellations in a paper he was writing. "As a courtesy, Coxeter sent Escher a copy of that paper, containing a figure of a hyperbolic tessellation" [3]. This new image intrigued Escher because it created a repeating pattern within a circle so that the repeating shapes became smaller as they moved toward the edge. Escher was quite skilled at compass and straightedge constructions and was able to easily repeat the construction of the hyperbolic tessellation, which eventually became his Circle Limit I piece.

As you can see in Figure 11, there is a connection between the 30-45-90 triangle tessellation and Circle Limit I. Whether working in the Euclidean plane or the hyperbolic plane, an artist can create a tessellation by modifying a base shape. If a section is removed from one side of the base shape, it must then be added to another side. In this case Escher most likely began with a kite formed by two adjacent triangles. He then removed small triangular pieces from each of the shorter sides of the kite and added them to the longer sides [3].

This print is a fabulous first attempt, but Escher had many criticisms of his own work. He said "both the form of the fishes, who have barely grown from rectilinear abstractions to rudimentary animals, and their placement with respect to one another 

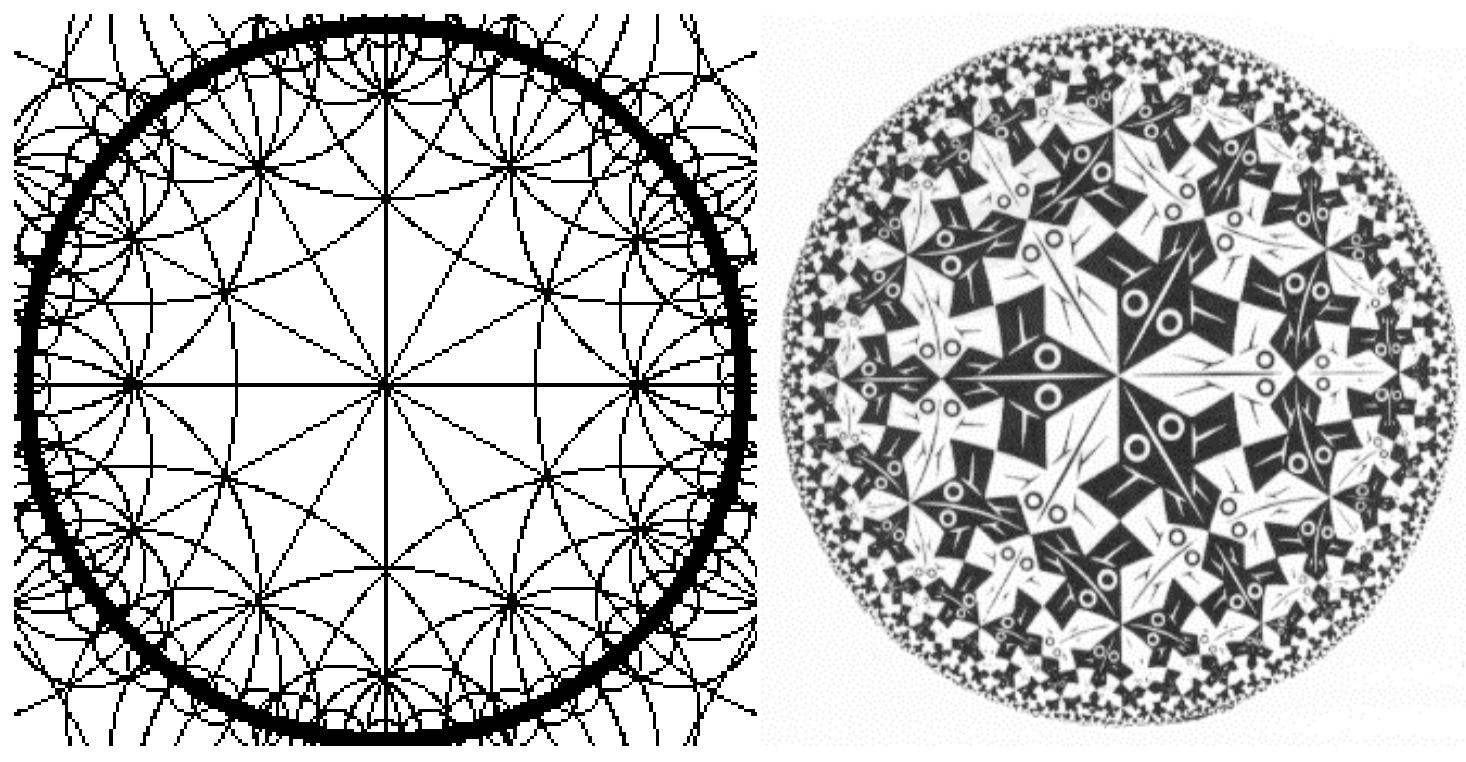

Figure 11. At left, 30-45-90 tiling; at right, M.C. Escher's Circle Limit I (right) @ 2004 The M.C. Escher Company - Baarn - Holland. All rights reserved.

leave something to be desired" [4]. He continues by criticizing the "traffic flow" of the fish: "They consist of white pairs with their heads turned to each other, alternating with black pairs whose tails touch each other. There is thus no continuity, no 'direction of the flow of traffic,' no unity of color in each of the rows" [4].

Circle Limit III repairs all of Escher's criticisms of Circle Limit $I$. This tiling is based on an $\{8,3\}$ tiling of the hyperbolic plane; one in which three octagons meet at each vertex. This print has been called the most stunning example of a hyperbolic tessellation, even though it really isn't a hyperbolic tessellation at all. For unknown reasons, the white lines along the backbones of the fish are not geodesics of the hyperbolic plane as they do not meet the boundary at right angles. In an article discussing Circle Limit III, Coxeter proved that the white lines meet the boundary at approximately 80 degrees, and this assertion can be confirmed by measuring the angles on the woodcut itself [2].

Circle Limit I and Circle Limit III are the two most recognized hyperbolic tessellation designs that Escher created. Escher's Circle Limit /I print is a hyperbolic tessellation but looks strikingly different than Circle Limit I and Circle Limit III. Escher didn't cease at the masterpiece of Circle Limit III; he concluded his series of hyperbolic tessellations with Circle Limit IV, also called Heaven and Hell (see Figure 12). This image is also based on the 3045-90 triangle tessellation that Escher employed in Circle Limit I. The drawbacks of Circle Limit I are not even noticeable in Circle Limit IV. Notice how Escher had mastered the art of eliminating the rectilinear

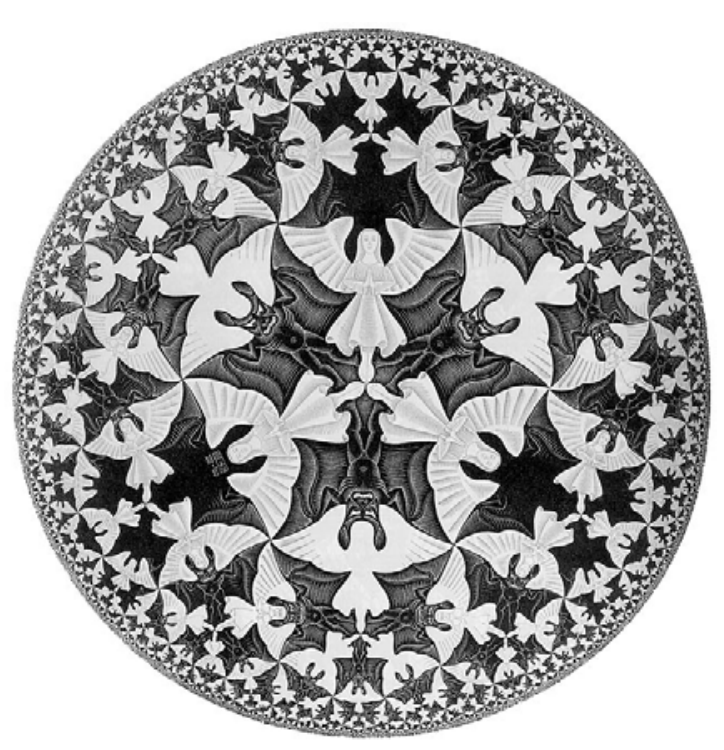

Figure 12. M.C. Escher's "Circle Limit IV" (C) 2004 The M.C. Escher Company Baarn - Holland. All rights reserved. 
edges that were so apparent in Circle Limit I. Because of this, it is more difficult to find the underlying tessellation, which makes the pattern much more mysterious and appealing.

\section{CLOSING REMARKS}

Hyperbolic space is often difficult to conceptualize because there are few natural models of surfaces that violate Euclid's fifth postulate. However, Poincaré's model and Escher's genius make hyperbolic geometry accessible to everyone. Tessellations are a great launching pad for investigating hyperbolic space. Even those without formal mathematical training can discover connections between Euclidean and hyperbolic space by looking at the tessellations of each plane as illustrated in the hyperbolic case by Escher's Circle Limit prints. Those with a mathematical background can delve deeper into the subject by investigating the isometries of hyperbolic space and the synthetic constructions of those isometries and tessellations.

Escher once said, "The ideas that are basic to [my works] often bear witness to my amazement and wonder at the laws of nature which operate in the world around us. $\mathrm{He}$ who wonders discovers that this is in itself a wonder. By keenly confronting the enigmas that surround us, and by considering and analyzing the observations that I had made, I ended up in the domain of mathematics. Although I am absolutely without training or knowledge in the exact sciences, I often seem to have more in common with mathematicians than with my fellow artists" [6].

\section{ACKNOWLEDGEMENT}

The authors thank the M.C. Escher Company for the rights to publish M.C. Escher's Circle Limit I, Circle Limit III, and Circle Limit IV (C) 2004 The M.C. Escher Company - Baarn - Holland. All rights reserved.

\section{REFERENCES}

1. Brannan, David A., Matthew F. Esplen, and Jeremy J. Gray. Geometry. (Cambridge University Press, Cambridge, UK, 1999).

2. Coxeter, H.S.M. "The Trigonometry of Escher's Woodcut Circle Limit III." Mathematical Intelligencer, Vol. 18, No. 4 (April 2003) pp. 42-46.

3. Dunham, Douglas. "A Tale Both Shocking and Hyperbolic." Math Horizons (April 2003) pp. 22-26.

4. Escher, M. C. Escher on Escher; Exploring the Infinite (Harry N. Abrams, Inc., New York, 1986).

5. Goodman-Strauss, Chaim. "Compass and Straightedge in the Poincaré Disk." The American Mathematical Monthly. Vol. 108, No. 1 (January 2001) pp. 3849.

6. Sarhangi, Reza. Artful Mathematics: "Review of M. C. Escher's Legacy: A Centennial Celebration." Notices of the AMS, Vol. 50, No. 4 (April 2003) pp. 45457.

7. Wallace, Edward C., and Stephen F. West, Roads to Geometry, Third Edition (Prentice-Hall, Upper Saddle River, New Jersey, USA, 2003).

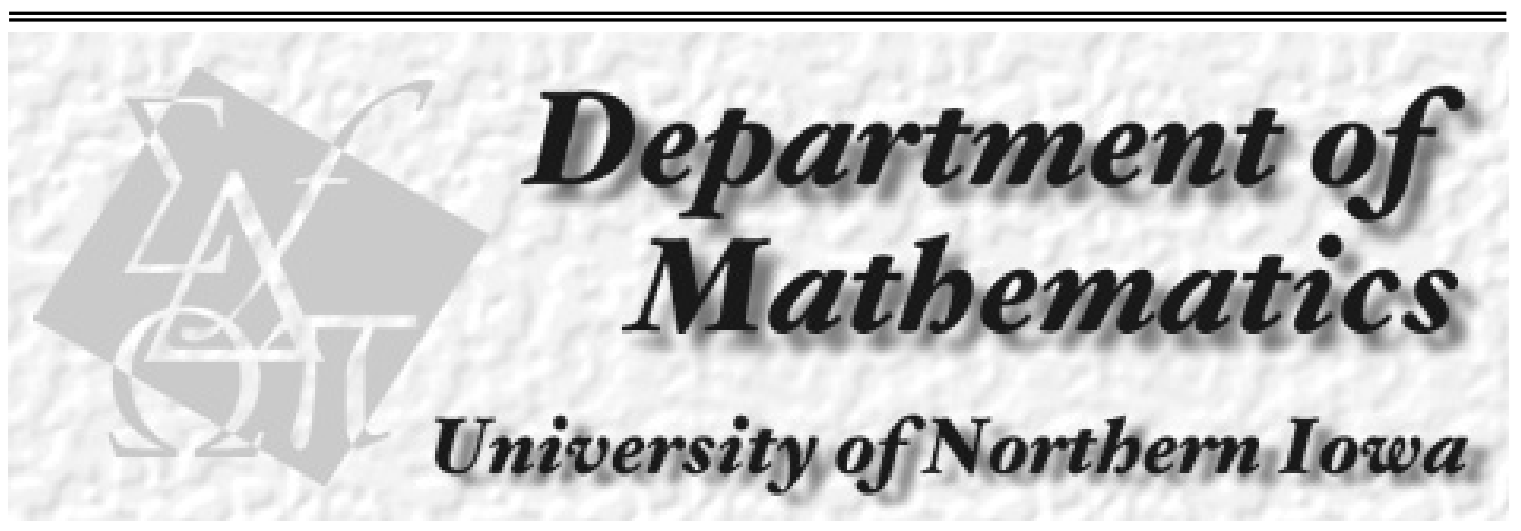

http://www.math.uni.edu/ 